\title{
Participation of the AngII/TRPC6/NFAT axis in the pathogenesis of podocyte injury in rats with type 2 diabetes
}

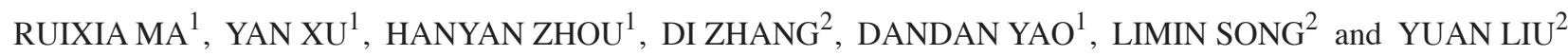 \\ ${ }^{1}$ Department of Nephrology, Affiliated Hospital of Qingdao University, Qingdao, Shandong 266000; \\ ${ }^{2}$ Department of Special Medicine, School of Medicine, Qingdao University, Qingdao, Shandong 266071, P.R. China
}

Received April 21, 2018; Accepted December 19, 2018

DOI: $10.3892 / \mathrm{mmr} .2019 .9871$

\begin{abstract}
The canonical transient receptor potential channel 6 ion channel is expressed in podocytes and is an important component of the glomerular slit diaphragm. Focal segmental glomerulosclerosis is closely associated with TRPC6 gene mutations, and TRPC6 mediates podocyte injury induced by high glucose. Angiotensin II (AngII) has been revealed to enhance TRPC6 currents in certain types of cells, including podocytes and ventricular myocytes. It has been reported that glucose regulated TRPC6 expression in an AngII-dependent manner in podocytes and that this pathway is critical in diabetic nephropathy. In the present study, the role of TRPC6 detected by western blotting and reverse transcription-quantitative polymerase chain reaction in AngII-mediated podocyte injury was evaluated in rats with type 2 diabetes induced by high-calorie diets and streptozotocin. The results demonstrated that urinary albumin excretion was elevated, and morphological changes, including glomerular basement membrane thickening and podocyte process effacement, were observed. There was increased expression of AngII and TRPC6 in diabetic rats. The angiotensin receptor blocker valsartan significantly reduced TRPC6 and nuclear factor of activated T-cells (NFAT) overexpression in diabetic rats. These results in vivo were confirmed by studies in vitro, which demonstrated that inhibition of TRPC6 ameliorated high glucose-induced podocyte injury by decreasing NFAT mRNA levels. Taken together, the present results suggested that the AngII/TRPC6/NFAT axis may be a crucial signaling pathway in podocytes that is necessary for maintaining the integrity of the glomerular filtration barrier. In addition, TRPC6 may represent a potential therapeutic target for diabetic nephropathy.
\end{abstract}

Correspondence to: Professor Ruixia Ma, Department of Nephrology, Affiliated Hospital of Qingdao University, 16 Jiangsu Road, Qingdao, Shandong 266000, P.R. China

E-mail: anita1685@163.com

Key words: diabetic nephropathy, podocyte, transient receptor potential channel 6, nuclear factor of activated T-cells, Angiotensin II

\section{Introduction}

Diabetic nephropathy has become the leading cause of end-stage renal disease worldwide, developing in $\sim 40 \%$ of patients with type 2 diabetes as glomerular function progressively declines. The glomerular filtration barrier consists of three main elements: Capillary endothelium, glomerular basement membrane (GBM) and a population of specialized cells (podocytes). In type 2 diabetes, mesangial expansion and GBM thickening are characteristics of renal dysfunction (1-3). In addition, alterations in podocyte structure, number and density are present at the onset of diabetic nephropathy $(4,5)$. In addition, podocyte effacement appears to contribute to microalbuminuria and to the pathogenesis of diabetic nephropathy (6-8).

Transient receptor potential (TRP) channels are a family of nonselective $\mathrm{Ca}^{2+}$-permeable cation channels that are widely expressed in vertebrate tissues. In particular, TRP channel 6 (TRPC6) expression and function have been studied in several tissues (9), including brain, kidney and heart (10-12). TRPC6 has been shown to serve an important role in the regulation of podocyte structure and function (13). Gain-of-function mutations in the TRPC6 gene resulted in hereditary focal segmental glomerulosclerosis (14), which is characterized by proteinuria, progressive renal failure and glomerular lesions. In addition, TRPC6 overexpression, which is associated with actin cytoskeleton rearrangement in podocytes, is a common feature of human proteinuric kidney diseases (10). TRPC6 knockdown by small interfering RNA (siRNA) attenuated high glucose-induced podocyte apoptosis, which contributes to the development of diabetic nephropathy (15). Sonneveld et al (16) reported that TRPC6 expression in podocytes was regulated by glucose in an Angiotensin II (AngII)-dependent manner (17). Taken together, these results suggested that TRPC6 is a potential therapeutic target for diabetic nephropathy. High glucose levels in diabetes increases AngII expression in glomeruli, especially in mesangial cells $(18,19)$. Ang-converting enzyme inhibitors and AngII receptor blockers (ARBs) have been shown to attenuate progressive glomerulosclerosis in several disease models (20-22). The ARB candesartan lowered the peak level of proteinuria by decreasing the expression of functional molecules in the slit diaphragm and slowed the progression of diabetic renal disease $(17,23)$. A recent study demonstrated that AngII perpetuated podocyte injury via 
the persistent activation of Notch1 and Snail signaling (24). These results demonstrate that AngII participates in mediating the effects of hyperglycemia in the progression of diabetic nephropathy (25).

Numerous studies have provided evidence for the involvement of AngII in the regulation of TRPC6. For example, AngII increased TPRC6 expression and activated TRPC6 currents in non-renal cells, including mesenteric artery myocytes and ventricular myocytes $(26,27)$. Accordingly, currents evoked by AngII in glomerular podocytes were eliminated by transfection with siRNA against TRPC6 (28). Increased TRPC6 expression in the podocyte membrane mediated apoptosis induced by AngII and albumin overload (29,30). TRPC6 also participated in the AngII-induced activation of nuclear factor in activated $\mathrm{T}$ cells (NFAT), contributing to the progression of cardiac hypertrophy (13). TRPC6-induced activation of the NFAT signaling pathway was identified as a potential mediator of focal segmental glomerulosclerosis (31). Furthermore, TRPC6-mediated $\mathrm{Ca}^{2+}$ influx and activation of $\mathrm{Ca}^{2+}$-dependent protein phosphatase calcineurin and its substrate NFAT have been implicated in nephropathy induced by doxorubicin and AngII (32). Nevertheless, it remains unclear as to whether AngII mediates podocyte changes associated with type 2 diabetic nephropathy via TRPC6.

It was hypothesized that high glucose levels result in podocyte injury through the activation of a pathway mediated by AngII, TRPC6 and NFAT. In the present study, it was revealed that increased AngII expression in glomerular podocytes was accompanied by enhanced urinary albumin excretion in a rat model of a high-calorie diet and streptozocin-induced type 2 diabetes. The ARB valsartan ameliorated diabetic podocyte injury via the downregulation of TRPC6 and NFAT. The results of the in vitro studies supported the role of TRPC6 and NFAT in AngII-induced podocyte injury.

\section{Materials and methods}

Animals. Pathogen-free male Wistar rats ( $\mathrm{n}=50$; 8-week-old; $200 \mathrm{~g}$ ) were obtained from the Institute of Drug Control (Qingdao, China). The rats were housed in individual cages in a temperature-controlled room with a 12-h light/dark cycle at 50-60\% relative humidity and were given food and water ad libitum. The rats were allowed to acclimatize for 1 week prior to the dietary intervention. Protocols for the animal experiments were approved by the Qingdao University Animal Care and Use Committee (Shandong, China) and were developed in accordance with guidelines set by the National Institutes of Health Guide for Care and Use of Laboratory Animals. The present study was approved by the Ethics Committee of the Affiliated Hospital of Qingdao University.

The rat model of type 2 diabetes was generated according to a previously described method (33). The rats were randomly divided into two groups according to diet: Regular rat chow $(\mathrm{n}=10$; normal control group) or high-calorie diet $(\mathrm{n}=40$; $10 \%$ animal fat, $20 \%$ cane sugar, $2.5 \%$ cholesterol, $1 \%$ cholate and $66.5 \%$ regular chow). Following 8 weeks, rats fed the high-calorie diet were intraperitoneally injected with $30 \mathrm{mgxkg}^{-1}$ streptozocin to induce type 2 diabetes. One week later, fasting blood glucose and insulin levels were measured, and the insulin sensitivity index was calculated as $22.5 /$ [fasting blood glucose (FBG) x insulin (INS)]. Rats with fasting blood glucose levels $>10.0 \mathrm{mmol} \cdot \mathrm{l}^{-1}$ and higher insulin levels $(\mathrm{n}=40)$ were further subdivided into two groups: The ARB treatment group $(n=20)$ receiving valsartan $(40 \mathrm{mg} / \mathrm{kgxday}$ given orally; Novartis International AG, Basel, Switzerland), and the diabetes mellitus (DM) control group $(n=20)$ receiving normal saline. These treatments were administered once a day for 12 weeks.

Following the 12-week treatment, the rats were weighed and urine samples were collected. The rats were then sacrificed, blood samples were obtained, and the kidneys were collected and weighed.

Determination of urinary albumin and creatinine concentrations. To evaluate albumin and creatinine excretion, 24-h urine samples were collected from the rats every 2 weeks during the 12-week treatment period. Albumin was measured using a turbidimetric immunoassay kit (Shibayagi Co., Ltd., Shibukawa, Japan). Creatinine was measured using an automatic biochemistry analyzer (model no. 7600-020; Hitachi, Ltd., Tokyo, Japan).

Evaluation of metabolic parameters. Blood samples were obtained from the tail vein once per week. FBG was determined using a glucometer (OneTouch ${ }^{\mathrm{TM}}$ SureStep ${ }^{\mathrm{TM}}$; LifeScan, Inc., Milpitas, CA, USA). Serum insulin levels and glycated hemoglobin (HbA1c) levels were determined by enzyme-linked immunosorbent assay (cat. nos. YJ-58700 and YJ-0021a, Aquatic Diagnostic Ltd., Stirling, Scotland).

Serum creatinine, urea nitrogen, total cholesterol, triglyceride, low-density lipoprotein and high-density lipoprotein levels were determined using an automatic biochemistry analyzer. Creatinine clearance was calculated using the following formula: Creatinine clearance=urine creatinine concentration/[serum creatinine concentration $\mathrm{x}$ volume of urine (ml) per min)].

Noninvasive blood pressure measurement. Blood pressure was measured using a tail cuff system (model LE5002; Diagnostic Systems Laboratories, Webster, TX, USA) in conscious rats, while animals rested in a climate-controlled room $\left(23^{\circ} \mathrm{C}\right)$. Systolic blood pressure was measured five times consecutively.

Glomerular morphological characteristics. Morphological characteristics of 50-nm renal cortex sections, including GBM thickness and the condition of podocyte processes, were examined and photographed using a JEM-1200 transmission electron microscope (JEOL, Ltd., Tokyo, Japan).

Cell culture. A conditionally immortalized mouse podocyte cell line, MPC5, was donated by The Central Laboratory of Shandong University (Shandong, China), and was cultured as previously described (34). The cell density at $60 \%$ were grown on plates in RPMI-1640 medium supplemented with $10 \%$ fetal bovine serum (cat. no. 1213G057, Beijing Solarbio Science \& Technology Co., Ltd., Beijing, China), 10-50 U/ml recombinant mouse $\gamma$-interferon (IFN), $100 \mathrm{U} / \mathrm{ml}$ penicillin and $100 \mathrm{mg} / \mathrm{ml}$ streptomycin at $33^{\circ} \mathrm{C}$ under a humidified atmosphere containing $5 \% \mathrm{CO}_{2}$. Then the conditions were changed to $37^{\circ} \mathrm{C}$ (without $\gamma$-IFN) to induce cell differentiation 
into mature podocytes and cells were cultured for 10-14 days. Differentiated podocytes were then divided into five groups: Normal glucose (NG) group (5.6 mmol/l), high glucose (HG) group (30 mmol/l), high mannitol group ( $\mathrm{NG}+$ mannitol $25 \mathrm{mmol} / \mathrm{l}$ ), the valsartan (VAL) group ( $\mathrm{HG}+$ the AngII receptor blocker VAR, $\left.10^{-5} \mathrm{mmol} / \mathrm{l}\right)$, and $\mathrm{HS}$ group $[\mathrm{HG}+$ the TRP channel inhibitor (35), SAR7334, $1 \mu \mathrm{M}$ ) cultured in $37^{\circ} \mathrm{C}$ for $48 \mathrm{~h}$.

Western blot analysis. Cultured podocytes or homogenized renal tissue were lysed in cold cell lysis buffer $(50 \mathrm{mM}$ Tris, $150 \mathrm{mM} \mathrm{NaCl}, 10 \mathrm{mM}$ ethylene diamine tetraacetic acid and $1 \%$ Triton $\mathrm{X}-100$ ) containing protease and phosphatase inhibitors. BCA protein assay kit (P0012, Beyotime Institute of Biotechnology, Haimen, China) and microplate reader (MD-SpectraMax, M5, USA) was used to determine total protein concentrations according to specification's instruction. The quantity of protein loaded per lane was $35 \mathrm{~g} / 101$. The proteins were separated by $10 \%$ sodium dodecyl sulfate-polyacrylamide gel electrophoresis and subsequently transferred to nitrocellulose membranes. Membranes were blocked with $3 \%$ nonfat dry milk for $2 \mathrm{~h}$ at room temperature. Primary antibodies against TRPC6 (sc-19196, goat anti-rat; 1:500), NFAT (sc-7296, mouse anti-rat; 1:2,000) and AngII (sc-20718, rabbit anti-rat; 1:1,000) were obtained from Santa Cruz Biotechnology, Inc. (Dallas, TX, USA) and incubated at $4^{\circ} \mathrm{C}$ overnight. Horseradish peroxidase-conjugated anti-immunoglobulin $\mathrm{G}$ served as the secondary antibody (ZB-2301, 1:5,000, Beijing Zhongshan Golden Bridge Biotechnology Co., Ltd.; OriGene Technologies, Inc., Beijing, China) and incubated at room temperature for $1 \mathrm{~h}$, and proteins were detected using an enhanced chemiluminescence kit (Beijing Zhongshan Golden Bridge Biotechnology Co., Ltd.; OriGene Technologies, Inc.) Image J (version 1.8.0, National Institutes of Health, Bethesda, MD, USA ) was used for intensity analysis.

Reverse transcription-quantitative polymerase chain reaction $(R T-q P C R)$. Total RNA was isolated from kidney cortex samples and cultured podocytes using TRIzol reagent (Invitrogen; Thermo Fisher Scientific, Inc., Waltham, MA, USA). The RNA was analyzed by $0.6 \%$ agarose gel electrophoresis, visualized by ethidium bromide and was quantified by spectrometry. Complementary cDNA was synthesized using PrimeScript RT Reagent Kit (cat. no. DRR037A; Takara Biotechnology Co., Ltd., Dalian, China). RT reactions were for $45 \mathrm{~min}$ at $25^{\circ} \mathrm{C}, 5 \mathrm{~min}$ at $85^{\circ} \mathrm{C}$ and then held at $4^{\circ} \mathrm{C}$. RT-qPCR was carried out using SYBR Premix Ex Taq (Takara Biotechnology Co., Ltd.) on an ABI PRISM 7000 HT (cat. no. 11744-100; Applied Biosystems; Thermo Fisher Scientific, Inc.) according to the manufacturer's protocol. Sequences of primers targeting TRPC6, AngII, and NFAT2 are shown in Table I. Reactions were incubated at $95^{\circ} \mathrm{C}$ for $30 \mathrm{sec}$, followed by 40 cycles of $95^{\circ} \mathrm{C}$ for $5 \mathrm{sec}, 60^{\circ} \mathrm{C}$ for $30 \mathrm{sec}$ and $72^{\circ} \mathrm{C}$ for $40 \mathrm{sec}$, followed by melting curve analysis. Relative gene expression was performed using the comparative $\mathrm{Cq}$ method $\left(2^{-\Delta \Delta \mathrm{Cq}}\right)(36)$ with GAPDH as the internal control.

Statistical analysis. Results were expressed as the mean \pm standard deviation of at least three independent experiments. Group differences were compared by one-way analysis of variance followed by Student-Newman-Keuls post hoc test. $\mathrm{P}<0.05$ was considered to indicate a statistically significant difference. Least significant different t-test was used for pairwise comparison, while Tamhane's T2 was used for Heteroscedasticity. All analyses were performed using SPSS software (version 22; IBM Corp., Armonk, IL, USA).

\section{Results}

Evaluation of metabolic parameters. The present study measured the metabolic parameters of rats in the normal control, DM and ARB treatment groups ( $n=10$ per group). The results revealed that there was a lower insulin sensitivity index, and increased levels of FBG, HbA1c and lipids in the DM group than in the normal controls (Table II). However, fasting insulin and serum creatinine levels did not differ between the groups. In addition, no significant differences in metabolic parameters were observed between the DM and ARB treatment groups (Table II).

Kidney function parameters. To assess glomerular injury, the present study measured urinary albumin excretion and found a significant difference between the DM and normal control groups. However, the ARB VAL significantly decreased urinary albumin excretion in the diabetic rats following 8 weeks of treatment (Fig. 1A). ARB treatment also decreased the elevated kidney weight/body weight ratio observed in diabetic rats (Fig. 1B) but did not affect creatinine clearance or systolic blood pressure (Fig. 1C and D).

AngII expression in glomerular podocytes and podocyte lesions in type 2 diabetic nephropathy. To better understand the role of AngII in podocytes, the present study analyzed AngII expression changes in the glomerular podocytes of type 2 diabetic rats via western blotting and qPCR. In addition, the effects of ARB treatment on GBM thickness and podocyte effacement were examined by electron microscopy. In the DM group, the mRNA and protein levels of AngII were significantly higher than those of the normal control group (Fig. 2A and B); however, treatment with VAL (40 mg/kgxday for 12 weeks) decreased AngII expression to levels almost equivalent to those of the normal controls. In DM rats, diabetic nephropathy was observed, which manifested as GBM thickening and podocyte process effacement (Fig. 2C). In addition, impairment of the glomerular filtration barrier was ameliorated by ARB treatment (Fig. 2).

Involvement of TRPC6 and NFAT in AngII-induced podocyte injury. To determine whether TRPC6 and NFAT were involved in AngII-induced podocyte injury in type 2 diabetes, the present study evaluated the expression of TRPC6 and NFAT in the rat model employed. The results revealed higher mRNA and protein levels of TRPC6 and NFAT in diabetic rats when compared with the normal controls (Fig. 3). These changes were attenuated by ARB treatment (Fig. 3).

ARB treatment attenuates $H G$-induced upregulation of AngII, TRPC6, and NFAT in vitro. As the results of the present study thus far suggested that the effects of AngII in the pathogenesis 
Table I. Primers used in the in vitro and in vivo studies.

A, In vitro studies using mouse podocytes

\begin{tabular}{lll}
\hline GenBank accession no. & \multicolumn{1}{c}{ Gene } & Sequence (5'-3') \\
\hline NM_001282086.1 & TRPC6 (F) & TCTCTGGTTTACGGCAGCAGA \\
& TRPC6 (R) & GGAGCTTGGTGCCTTCAAATC \\
NM_00164109.1 & NFAT2 (F) & GGTGCCTTTTGCGAGCAGTA \\
& NFAT2 (R) & TGAGCCCTGTGGTGAGACTTG \\
NM_001161731.2 & AngII (F) & ACTGCGAAAGTATGATGGTGAA \\
& AngII (R) & CCTTGATGTTGTTCTTGGTGTC \\
NM_001289726.1 & GAPDH (F) & CTCATGACCACAGTCCATGC \\
& GAPDH (R) & CACATTGGGGGTAGGAACAC
\end{tabular}

$\mathrm{B}$, In vivo studies in rats

\begin{tabular}{lll}
\hline GenBank accession no. & Gene & Sequence (5'-3') \\
\hline NM_053559.1 & TRPC6 (F) & TACGGATTGTGGAGGCTATTCT \\
& TRPC6 (R) & AAAGTCATCTTGCTGGAGTTCA \\
NM_001244933.1 & NFAT2 (F) & GAGGGAAGAAGATGGTGTTGTC \\
& NFAT2 (R) & GCACAGGTCTCGGTCAGTTT \\
NM_001006992.1 & AngII (F) & GCAAGCATACAGGAGGGTCTC \\
& AngII (R) & CCATTCTCACAGGCAATAACAA \\
NM_001289726.1 & GAPDH (F) & CTCATGACCACAGTCCATGC \\
& GAPDH (R) & CACATTGGGGGTAGGAACAC \\
\hline
\end{tabular}

TRPC6, transient receptor potential channel 6; NFAT, nuclear factor of activated T-cells; AngII, angiotensin II; F, forward; R, reverse.

Table II. Biochemical parameters in diabetic rats following 12-week treatment with the angiotensin II receptor blocker, valsartan.

\begin{tabular}{lccr}
\hline Characteristics & NC $(\mathrm{n}=10)$ & $\mathrm{DM}(\mathrm{n}=10)$ & ARB $(\mathrm{n}=10)$ \\
\hline ISI & $0.220 \pm 0.024$ & $0.05 \pm 0.004^{\mathrm{a}}$ & $0.060 \pm 0.005^{\mathrm{a}}$ \\
FINS ng/ml & $18.57 \pm 1.01$ & $22.09 \pm 1.75$ & $20.16 \pm 1.57$ \\
FBG mmol/1 & $5.56 \pm 0.64$ & $19.44 \pm 0.47^{\mathrm{a}}$ & $18.58 \pm 0.58^{\mathrm{a}}$ \\
$\mathrm{HbAlC} \%$ & $2.84 \pm 0.33$ & $6.64 \pm 0.45^{\mathrm{a}}$ & $6.40 \pm 0.72^{\mathrm{a}}$ \\
TG mmol/1 & $1.49 \pm 0.15$ & $4.83 \pm 0.69^{\mathrm{a}}$ & $4.42 \pm 0.48^{\mathrm{a}}$ \\
TC mmol/1 & $0.76 \pm 0.24$ & $3.99 \pm 0.25^{\mathrm{a}}$ & $3.78 \pm 0.26^{\mathrm{a}}$ \\
LDL mmol/1 & $1.04 \pm 0.19$ & $7.38 \pm 0.44^{\mathrm{a}}$ & $7.07 \pm 0.45^{\mathrm{a}}$ \\
HDL mmol/l & $1.03 \pm 0.20$ & $0.50 \pm 0.04^{\mathrm{a}}$ & $0.52 \pm 0.04^{\mathrm{a}}$ \\
Scr mmol/l & $48.5 \pm 7.09$ & $53.7 \pm 7.83$ & $50 \pm 8.84$ \\
\hline
\end{tabular}

${ }^{\mathrm{a}} \mathrm{P}<0.01$ vs. NC. Results are expressed as mean \pm standard deviation. ISI, insulin sensitivity index; FINS, fasting serum insulin, FBG: fasting blood glucose; HbA1C, glycosylated hemoglobin; TC, total cholesterol; TG, triglyceride; LDL, low-density lipoprotein cholesterol; HDL, high-density lipoprotein cholesterol; Scr, serum creatinine; NC, normal control; DM, rat model of type 2 diabetes mellitus; $\mathrm{ARB}$, angiotensin II receptor blocker (rats with type 2 diabetes treated with angiotensin II receptor blocker, valsartan).

of diabetic nephropathy were mediated by TRPC6/NFAT signaling, the present study then investigated whether this pathway was activated by $\mathrm{HG}$ levels in cultured podocytes. The protein levels (Fig. 4A-C) and mRNA levels (Fig. 4D-F) of AngII, TRPC6, and NFAT were markedly higher in cells exposed to HG (30 mmol/l) for $48 \mathrm{~h}$ when compared with cells exposed to NG levels $(5.6 \mathrm{mmol} / \mathrm{l})$. This effect was significantly attenuated by ARB treatment with VAL. Cells cultured with $5.6 \mathrm{mmol} / 1$ glucose and $25 \mathrm{mmol} / 1 \mathrm{mannitol}$ were also evaluated. The expression of AngII, TRPC6 and NFAT in these cells was similar to that of the NG control group, thereby ruling out the osmotic effect of glucose. 

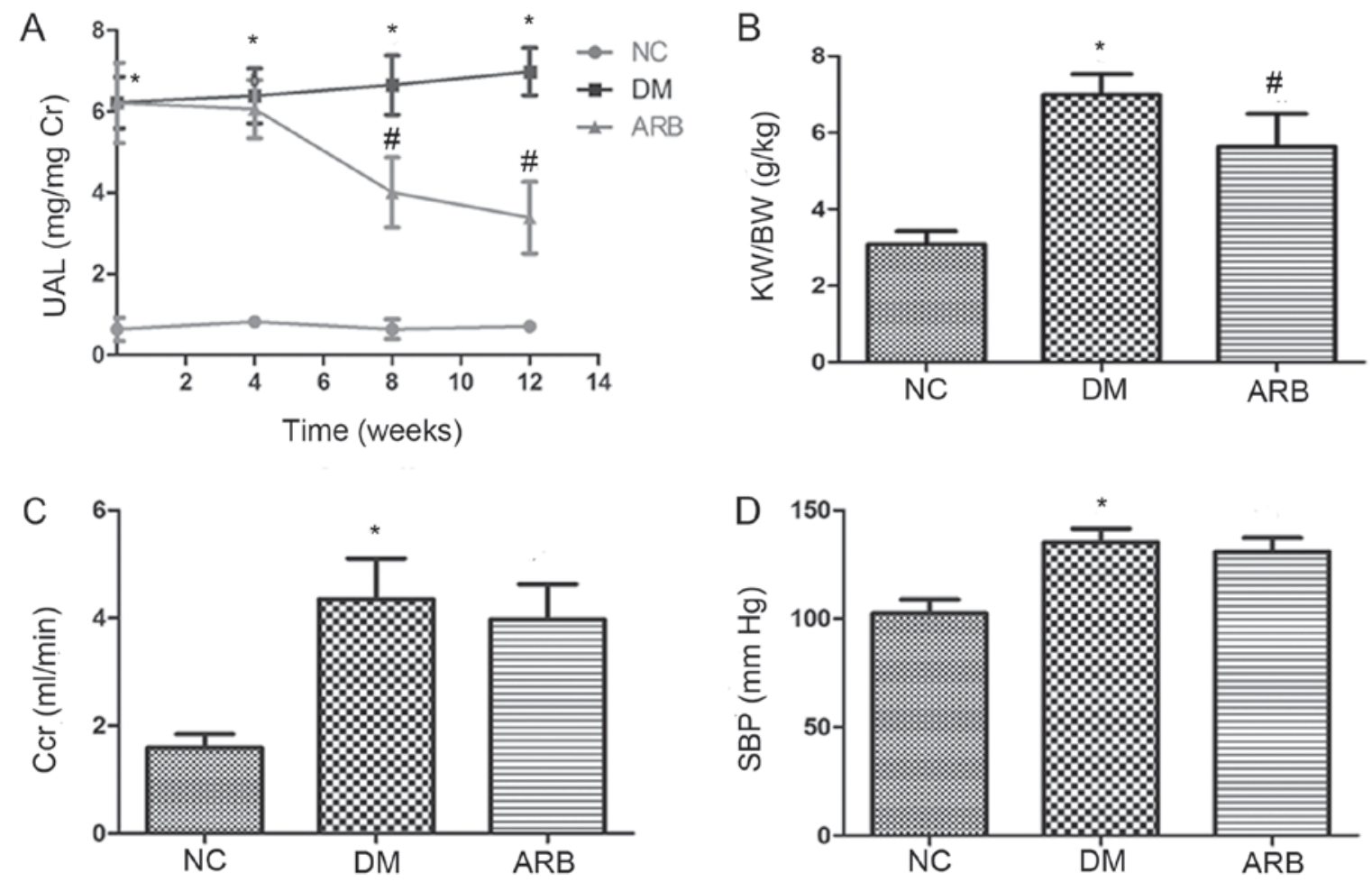

Figure 1. Effect of angiotensin II receptor blocker valsartan on functional parameters of diabetic rats. (A) UAL was elevated in rats with streptozocin-induced DM when compared with NC; however, albumin excretion was decreased in rats treated with the ARB, valsartan. (B) Valsartan also decreased the ratio of KW/BW in diabetic rats, but did not alter the (C) Ccr or (D) SBP. "P $<0.01$ vs. NC group; ${ }^{\#} \mathrm{P}<0.01$ vs. DM group. UAL, urinary albumin level; DM, diabetes mellitus; NC, normal control; ARB, Angiotensin II receptor blocker; KW/BW, kidney weight to body weight; Ccr, creatinine clearance; SBP, systolic blood pressure.
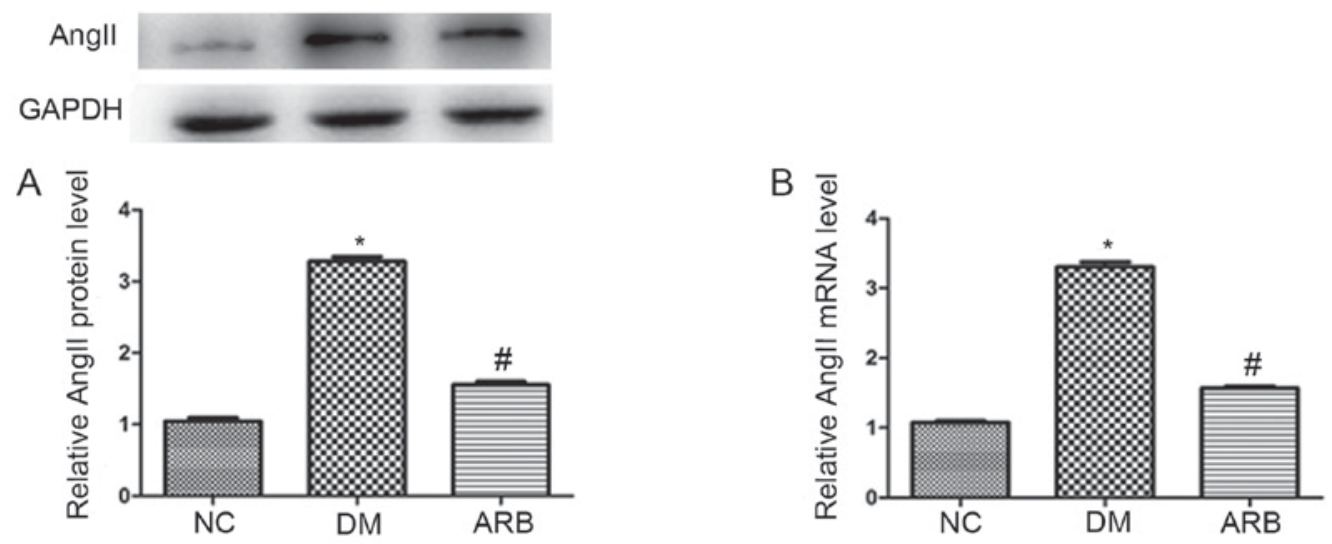

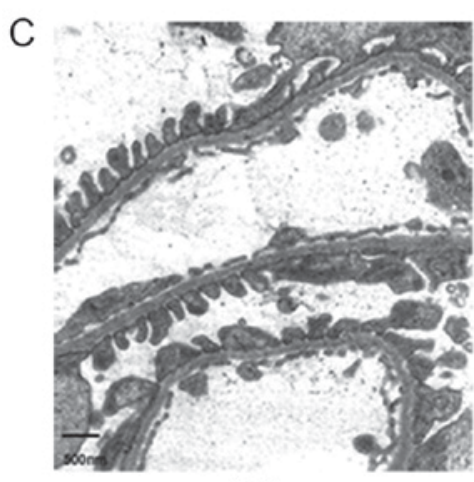

NC

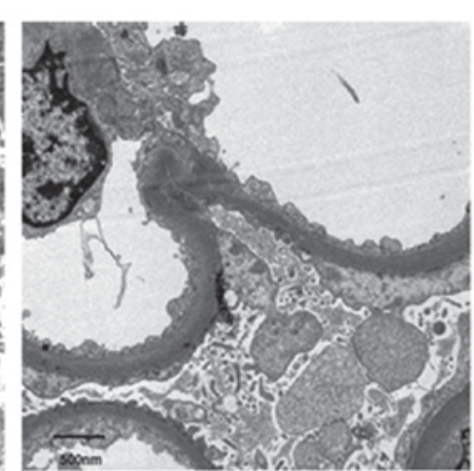

$\mathrm{DM}$

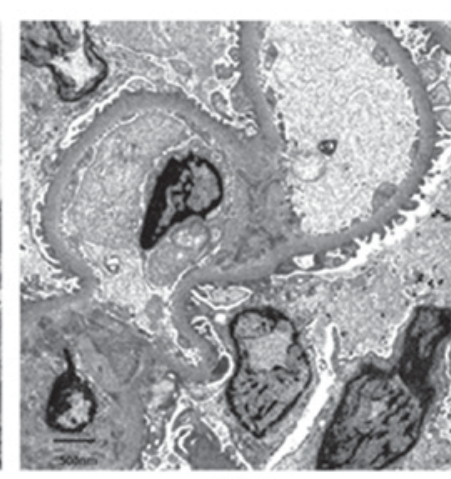

ARB

Figure 2. Effect of the ARB valsartan on nephropathy. AngII expression was attenuated in the kidneys of diabetic rats treated with the ARB valsartan when compared with that of untreated DM rats, at the (A) protein and (B) mRNA levels. (C) Valsartan also attenuated glomerular basement membrane thickening and podocyte process effacement. NC rats $(n=10)$ were fed regular rat chow, whereas rats in the $\mathrm{DM}(\mathrm{n}=10)$ and ARB groups $(\mathrm{n}=10)$ were fed a high-calorie diet and injected with streptozocin. The ARB group also received $40 \mathrm{mg} / \mathrm{kgxday}$ valsartan once a day for 12 weeks. "P<0.01 vs. NC group; ${ }^{*} \mathrm{P}<0.01 \mathrm{vs}$. DM group. AngII, angiotensin II; ARB, angiotensin II receptor blocker; DM, diabetes mellitus; NC, normal control. 

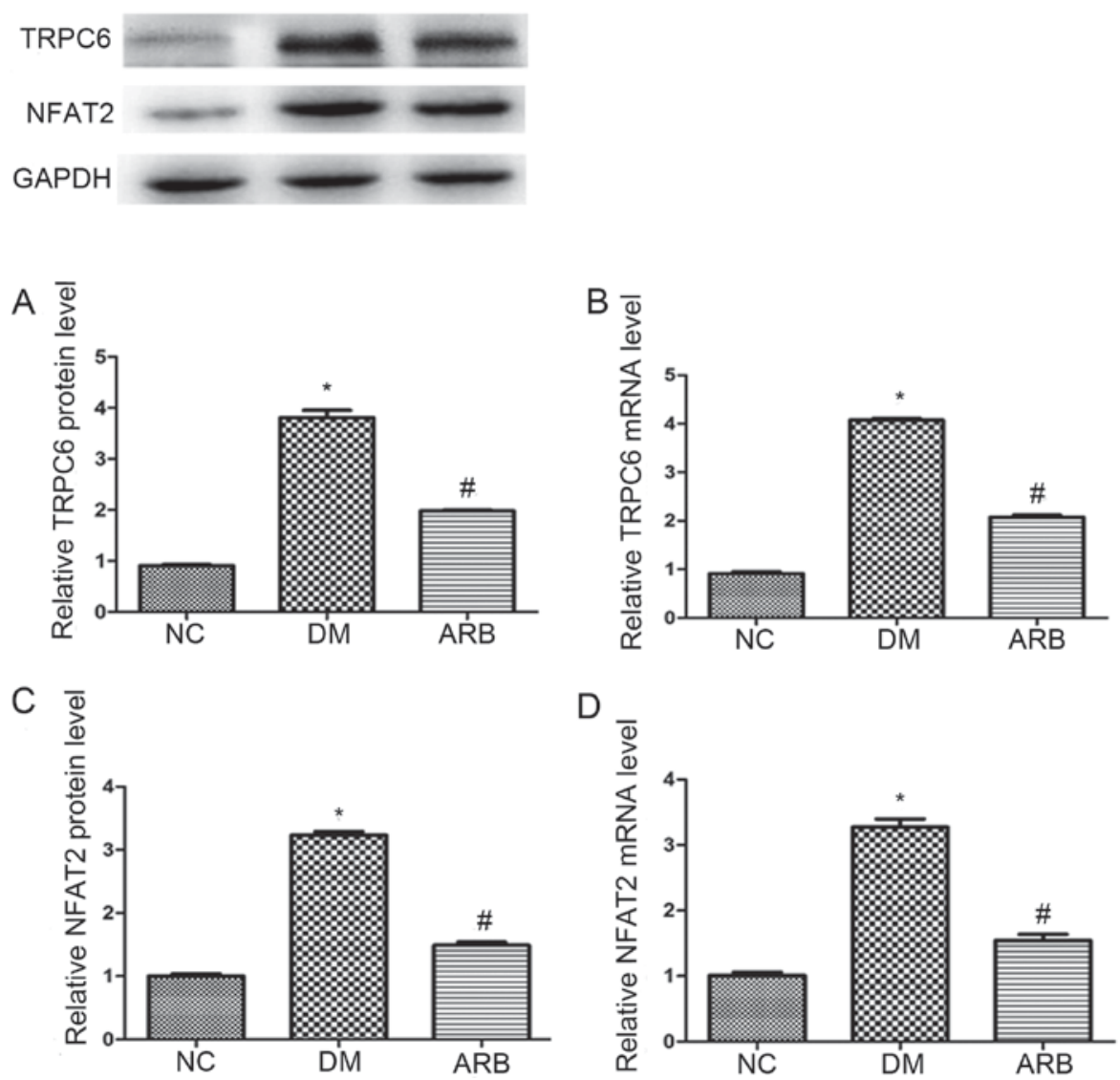

Figure 3. AngII upregulates TRPC6 and NFAT2 expression. Expression of TRPC6 and NFAT2 was attenuated in the kidneys of diabetic rats treated with valsartan (ARB group) when compared with that of untreated DM rats, at the (A and C) protein and (B and D) mRNA levels. NC rats ( $\mathrm{n}=10)$ were fed regular rat chow, whereas rats in the DM $(n=10)$ and ARB groups $(n=10)$ were fed a high-calorie diet and injected with streptozocin. The ARB group also received $40 \mathrm{mg} / \mathrm{kgxday}$ valsartan once a day for 12 weeks. ${ }^{\mathrm{P}}<0.01$ vs. NC group; ${ }^{*} \mathrm{P}<0.01 \mathrm{vs.}$. DM group. AngII, angiotensin II; ARB, angiotensin II receptor blocker; DM, diabetes mellitus; NC, normal control; TRPC6, transient receptor potential channel 6; NFAT, nuclear factor of activated T-cells.

TRP channel inhibitor attenuates $H G$-induced NFAT overexpression in vitro. To further study the role of TRPC6 in this signaling pathway, the present study evaluated the effect of the TRP channel inhibitor SAR7334 on TRPC6 and NFAT expression in cultured podocytes. The results revealed that SAR7334 (the HS group) significantly attenuated the HG-induced increase of AngII, TRPC6 and NFAT expression, at the protein (Fig. 5A-C) and mRNA levels (Fig. 5D-F).

\section{Discussion}

Previous studies have reported significantly decreased podocyte numbers and density in patients with diabetic nephropathy, as well as a negative correlation between proteinuria and podocyte injury (37). These investigations contributed greatly to our understanding of the role podocyte injury serves in the glomerular dysfunction of diabetic nephropathy $(38,39)$. Identifying mechanisms underlying these podocyte structural and functional changes may provide novel therapeutic targets for diabetic nephropathy. The present study revealed that treatment with the ARB VAL had no significant effect on systolic blood pressure or creatinine clearance at the administered dose, suggesting that VAL reduced proteinuria by protecting against podocyte injury.

Podocytes in the kidneys as well as in endothelial and distal tubular cells express TRPC6 (40-42). To further explore this phenomenon, the present study measured TRPC6 expression in cultured podocytes. The results revealed the increase in AngII and TRPC6 in the kidneys of diabetic rats and HG-treated podocytes were consistent with those of studies by Durvasula and Shankland (43) and Yoo et al (44) who reported that exposure to HG levels increased AngII and its receptor AngII receptor type 1 in podocytes. Other studies have demonstrated that increased renin secretion and prorenin receptor activity contributed to the activation of the local angiotensin system in a HG environment, driving podocyte injury and loss. In an attempt to further elucidate the mechanisms underlying AngII-induced podocyte injury in diabetic nephropathy, the present study investigated the role of TRPC6 and its substrate NFAT. NFAT is a transcription factor that is predominantly regulated by activation and the subsequent translocation from the cytoplasm to the nucleus. NFAT activation/regulation is measured by total NFAT2 protein/mRNA expression levels $(45,46)$. The results demonstrated that the ARB VAL attenuated the HG-induced increase of TRPC6 and NFAT in podocytes in vivo and in vitro. In addition, changes in albumin excretion and glomerular morphology were ameliorated by ARB treatment. In particular, VAL reversed foot process loss and injury, resulting in a near-normal appearance. This provided further evidence of the role of TRPC6 in AngII-induced podocyte dysfunction. Recent studies have reported 


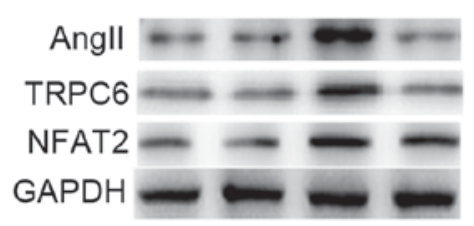

A

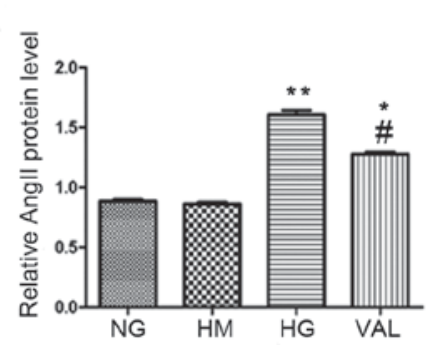

D

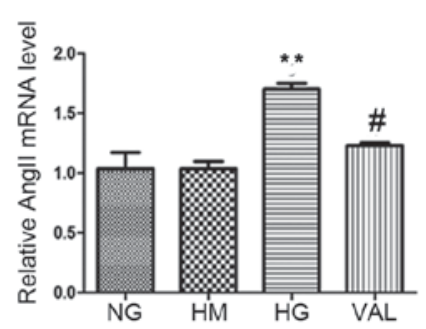

B

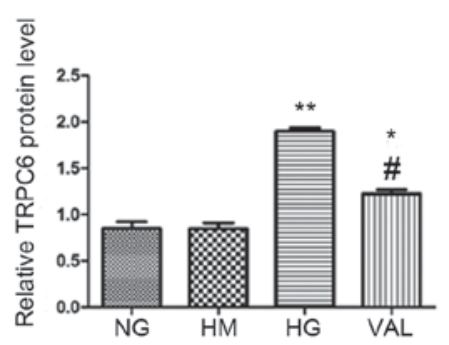

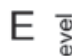

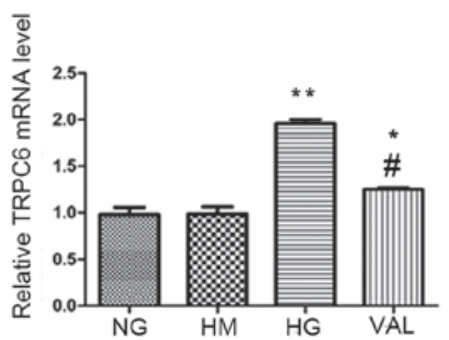

C

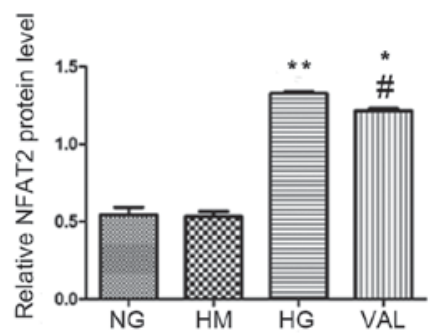

$\mathrm{F}$

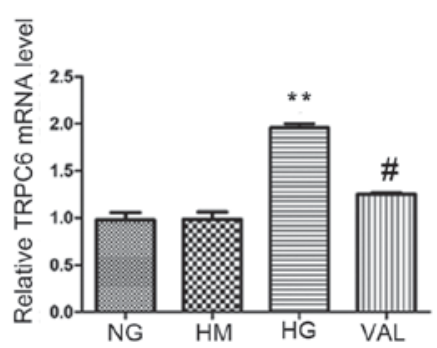

Figure 4. ARB VAL attenuates the HG-induced upregulation of AngII, TRPC6 and NFAT in vitro. Expression of AngII, TRPC6 and NFAT was assessed by (A-C) western blotting and (D-F) reverse transcription-quantitative polymerase chain reaction analysis in podocytes cultured with $\mathrm{NG}$ (5.6 mmol/l) or HG ( $30 \mathrm{mmol} / \mathrm{l})$. Podocytes cultured with NG and HM $(25 \mathrm{mmol} / \mathrm{l})$ served as a control for the effects of osmotic pressure. The effect of the ARB was evaluated in podocytes cultured with $\mathrm{HG}(30 \mathrm{mmol} / \mathrm{l})$ and treated with VAL $(10.5 \mathrm{mmol} / \mathrm{l}) .{ }^{*} \mathrm{P}<0.05$ and ${ }^{* *} \mathrm{P}<0.01$ vs. NG group; ${ }^{*} \mathrm{P}<0.05$ vs. HG group. AngII, angiotensin II; ARB, angiotensin II receptor blocker; NG, normal glucose; HG, high glucose; HM, high mannitol; VAL, valsartan; TRPC6, transient receptor potential channel 6; NFAT, nuclear factor of activated T-cells.
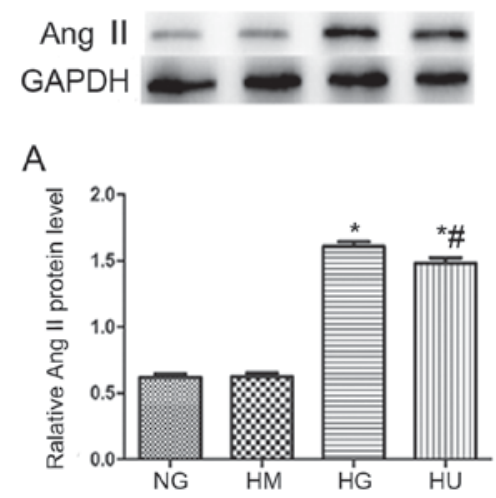

$\mathrm{D}$

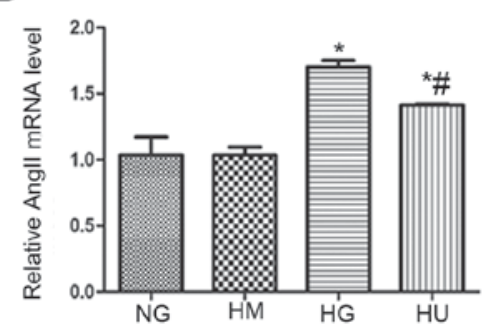

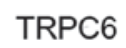
GAPDH

B

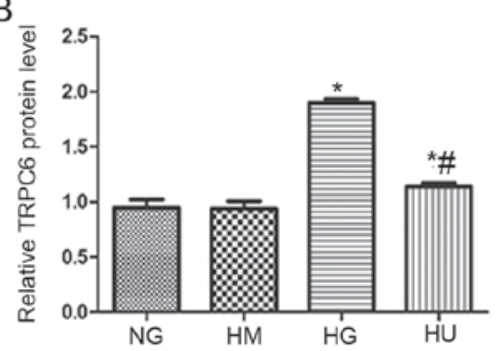

E

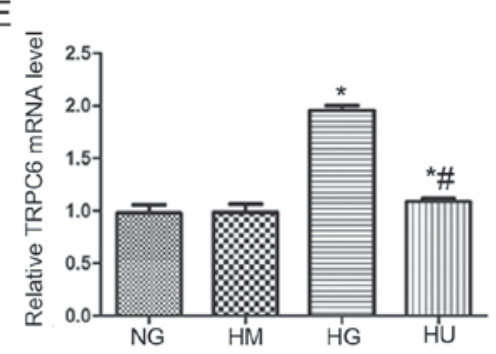

NFAT2

GAPDH

C

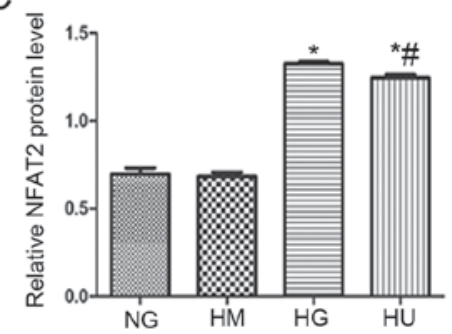

F

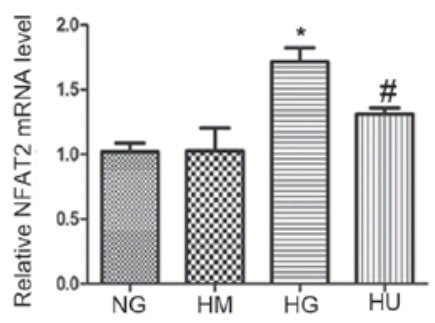

Figure 5. TRPC inhibitor SAR7334 attenuates the HG-induced upregulation of TRPC6 and NFAT. Expression of AngII, TRPC6 and NFAT was assessed by (A-C) western blotting and (D-F) reverse transcription-quantitative polymerase chain reaction analysis in podocytes cultured with NG (5.6 mmol/1) or HG (30 mmol/1). Podocytes cultured with NG and HM (25 mmol/l) served as a control for the effects of osmotic pressure. The effects of TRPC inhibition were evaluated in podocytes cultured with HG $(30 \mathrm{mmol} / \mathrm{l})$ and treated with SAR7334 (the HS group; $1 \mu \mathrm{M})$. ${ }^{*} \mathrm{P}<0.05 \mathrm{vs}$. NG group; ${ }^{*} \mathrm{P}<0.05$ vs. HG group. TRPC6, transient receptor potential channel 6; NFAT, nuclear factor of activated T-cells; NG, normal glucose; HG, high glucose; HM, high mannitol; HS, high glucose + the TRP channel inhibitor, SAR7334; AngII, angiotensin II. 
that TRPC6 was closely associated with HG-induced apoptosis in cultured podocytes through reactive oxygen species production and the RhoA/Rho-associated protein kinase signaling pathway $(15,47)$. In addition, Li et al (48) described the involvement of the canonical Wnt signaling pathway in diabetic podocyte injury caused by TRPC6 upregulation. However, Liu et al (15) and Yang et al (47) did not confirm their results in vivo. In the present study, a rat model of a high-calorie diet and streptozocin-induced type 2 diabetes was employed to study the role of TRPC6 in podocyte lesions associated with diabetic nephropathy.

Several lines of evidence have suggested that there is a close association between AngII and TRPC6 in non-renal and glomerular cells. For example, TRPC6 has been implicated in AngII-induced vasoconstrictor responses in mesenteric artery myocytes (27). Furthermore, the ARB losartan reversed the effect of AngII on glomerular mesangial cell proliferation by decreasing TRPC6 expression (49). Other cell culture and animal studies demonstrated that AngII had a deleterious effect on podocytes via the upregulation of TRPC6 (32), and that this process required the generation of reactive oxygen species (28). The present results support our hypothesis that TRPC6 is a key mediator of AngII-induced podocyte injury in the progression of type 2 diabetic nephropathy.

To further evaluate the downstream signaling pathway of AngII in podocytes, the present evaluated the effect of high glucose conditions on the expression of NFAT, a substrate for calcineurin. Other studies have reported that HG levels activated NFAT in vascular smooth muscle and pancreatic $\beta$-cells $(50,51)$. Furthermore, NFAT activation was involved in podocyte injury and glomerulosclerosis, and NFAT mediated HG-induced glomerular podocyte apoptosis through the upregulation of B-cell lymphoma 2 -associated X protein (Bax) $(31,52,53)$. Zhang et al (54) recently demonstrated that an NFAT inhibitor exerted renoprotective effects in diabetic $\mathrm{db} / \mathrm{db}$ rats by attenuating HG-induced podocyte apoptosis.

The present study revealed that HG-induced NFAT levels were attenuated by treatment with the TRPC6 channel inhibitor SAR7334 in podocytes, suggesting that hyperglycemia activated TRPC6 channels via increased AngII expression. The augmented $\mathrm{Ca}^{2+}$ influx caused the activation of NFAT, mediating podocyte apoptosis by increasing Bax expression, leading to glomerular filtration barrier dysfunction.

In conclusion, the results of the present study suggest that the AngII/TRPC6/NFAT axis may mediate podocyte injury in the early progression of type 2 diabetic nephropathy. In addition, TRPC6 may represent a novel therapeutic target for diabetic nephropathy.

\section{Acknowledgements}

The authors would like to thank Professor Liqiu Liu (Department of Nephrology, Affiliated Hospital of Qingdao University, Qingdao, China) for providing the conditionally immortalized mouse podocyte cell line used in the present study, as well as Professor Donghui Zhen (Department of Pathology, Qilu Hospital of Shandong University, Qingdao, China)for assisting with electron microscopy.

\section{Funding}

No funding was received.

\section{Availability of data and materials}

The datasets used and/or analyzed during the current study are available from the corresponding author on reasonable request.

\section{Authors' contributions}

RM designed and coordinated the present study, and performed the experiments. RM, YX, HZ, DZ, DY, LS and YL analyzed the data. RM, LS and YL wrote the paper.

\section{Ethics approval and consent to participate}

Protocols for the animal experiments were approved by the Qingdao University Animal Care and Use Committee (Qingdao, China) and were developed in accordance with guidelines set by the National Institutes of Health Guide for Care and Use of Laboratory Animals. The present study was approved by the Ethics Committee of the Affiliated Hospital of Qingdao University.

\section{Patient consent for publication}

Not applicable.

\section{Competing interests}

The authors declare that they have no competing interests.

\section{References}

1. Fioretto P, Mauer M, Brocco E, Velussi M, Frigato F, Muollo B, Sambataro M, Abaterusso C, Baggio B, Crepaldi G and Nosadini R: Patterns of renal injury in NIDDM patients with microalbuminuria. Diabetologia 39: 1569-1576, 1996.

2. Osterby R, Gall MA, Schmitz A, Nielsen FS, Nyberg G and Parving HH: Glomerular structure and function in proteinuric type 2 (non-insulin-dependent) diabetic patients. Diabetologia 36: 1064-1070, 1993.

3. White KE and Bilous RW: Type 2 diabetic patients with nephropathy show structural-functional relationships that are similar to type 1 disease. J Am Soc Nephrol 11: 1667-1673, 2000.

4. Dalla Vestra M, Masiero A, Roiter AM, Saller A, Crepaldi G and Fioretto P: Is podocyte injury relevant in diabetic nephropathy? Studies in patients with type 2 diabetes. Diabetes 52: 1031-1035, 2003.

5. White KE, Bilous RW and Diabiopsies Study Group: Structural alterations to the podocyte are related to proteinuria in type 2 diabetic patients. Nephrol Dial Transplantat 19: 1437-1440, 2004.

6. Meyer TW, Bennett PH and Nelson RG: Podocyte number predicts long-term urinary albumin excretion in Pima Indians with Type II diabetes and microalbuminuria. Diabetologia 42: 1341-1344, 1999.

7. Nakamura T, Ushiyama C, Osada S, Hara M, Shimada N and Koide H: Pioglitazone reduces urinary podocyte excretion in type 2 diabetes patients with microalbuminuria. Metabolism 50: 1193-1196, 2001.

8. Pagtalunan ME, Miller PL, Jumping-Eagle S, Nelson RG, Myers BD, Rennke HG, Coplon NS, Sun L and Meyer TW: Podocyte loss and progressive glomerular injury in type II diabetes. J Clin Invest 99: 342-348, 1997.

9. Estacion M, Sinkins WG, Jones SW, Applegate MA and Schilling WP: Human TRPC6 expressed in HEK 293 cells forms non-selective cation channels with limited $\mathrm{Ca} 2+$ permeability. J Physiol 572: 359-377, 2006. 
10. Möller CC, Wei C, Altintas MM, Li J, Greka A, Ohse T, Pippin JW, Rastaldi MP, Wawersik S, Schiavi S, et al: Induction of TRPC6 channel in acquired forms of proteinuric kidney disease. J Am Soc Nephrol 18: 29-36, 2007.

11. Onohara N, Nishida M, Inoue R, Kobayashi H, Sumimoto H, Sato Y, Mori Y, Nagao T and Kurose H: TRPC3 and TRPC6 are essential for angiotensin II-induced cardiac hypertrophy. EMBO J 25: 5305-5316, 2006.

12. Tai Y, Feng S, Ge R, Du W, Zhang X, He Z and Wang Y: TRPC6 channels promote dendritic growth via the CaMKIV-CREB pathway. J Cell Sci 121: 2301-2307, 2008.

13. Reiser J, Polu KR, Möller CC, Kenlan P, Altintas MM, Wei C, Faul C, Herbert S, Villegas I, Avila-Casado C, et al: TRPC6 is a glomerular slit diaphragm-associated channel required for normal renal function. Nat Genet 37: 739-744, 2005.

14. Winn MP, Conlon PJ, Lynn KL, Farrington MK, Creazzo T, Hawkins AF, Daskalakis N, Kwan SY, Ebersviller S Burchette JL, et al: A mutation in the TRPC6 cation channel causes familial focal segmental glomerulosclerosis. Science 308: 1801-1804, 2005

15. Liu BC, Song X, Lu XY, Li DT, Eaton DC, Shen BZ, Li XQ and Ma HP: High glucose induces podocyte apoptosis by stimulating TRPC6 via elevation of reactive oxygen species. Biochim Biophys Acta 1833: 1434-1442, 2013.

16. Sonneveld R, van der Vlag J, Baltissen MP, Verkaart SA, Wetzels JF, Berden JH, Hoenderop JG and Nijenhuis T: Glucose specifically regulates TRPC6 expression in the podocyte in an AngII-dependent manner. Am J Pathol 184: 1715-1726, 2014.

17. Brenner BM, Cooper ME, de Zeeuw D, Keane WF, Mitch WE, Parving HH, Remuzzi G, Snapinn SM, Zhang Z, Shahinfar S and RENAAL Study Investigators: Effects of losartan on renal and cardiovascular outcomes in patients with type 2 diabetes and nephropathy. N Engl J Med 345: 861-869, 2001.

18. Singh R, Singh AK and Leehey DJ: A novel mechanism for angiotensin II formation in streptozotocin-diabetic rat glomeruli. Am J Physiol Renal Physiol 288: F1183-F1190, 2005.

19. Vidotti DB, Casarini DE, Cristovam PC, Leite CA, Schor N and Boim MA: High glucose concentration stimulates intracellular renin activity and angiotensin II generation in rat mesangial cells. Am J Physiol Renal Physiol 286: F1039-F1045, 2004.

20. Bansal SB, Sethi SK, Jha P, Duggal R and Kher V: Remission of post-transplant focal segmental glomerulosclerosis with angiotensin receptor blockers. Indian J Nephrol 27: 154-156, 2017.

21. Hamaguchi A, Kim S, Wanibuchi $\mathrm{H}$ and Iwao H: Angiotensin II and calcium blockers prevent glomerular phenotypic changes in remnant kidney model. J Am Soc Nephrol 7: 687-693, 1996.

22. Hayashi K, Sasamura H, Ishiguro K, Sakamaki Y, Azegami T and Itoh H: Regression of glomerulosclerosis in response to transient treatment with angiotensin II blockers is attenuated by blockade of matrix metalloproteinase-2. Kidney Int 78: 69-78, 2010.

23. Suzuki K, Han GD, Miyauchi N, Hashimoto T, Nakatsue T, Fujioka Y, Koike H, Shimizu F and Kawachi H: Angiotensin II type 1 and type 2 receptors play opposite roles in regulating the barrier function of kidney glomerular capillary wall. Am J Pathol 170: 1841-1853, 2007.

24. Gagliardini E, Perico N, Rizzo P, Buelli S, Longaretti L, Perico L, Tomasoni S, Zoja C, Macconi D, Morigi M, et al: Angiotensin II contributes to diabetic renal dysfunction in rodents and humans via Notch1/Snail pathway. Am J Pathol 183: 119-130, 2013.

25. Leehey DJ, Singh AK, Alavi N and Singh R: Role of angiotensin II in diabetic nephropathy. Kidney Int (Suppl 77): S93-S98, 2000.

26. Kuwahara K, Wang Y, McAnally J, Richard son JA, Bassel-Duby R, Hill JA and Olson EN: TRPC6 fulfills a calcineurin signaling circuit during pathologic cardiac remodeling. J Clin Invest 116: 3114-3126, 2006.

27. Saleh SN, Albert AP, Peppiatt CM and Large WA: Angiotensin II activates two cation conductances with distinct TRPC1 and TRPC6 channel properties in rabbit mesenteric artery myocytes. J Physiol 577: 479-495, 2006.

28. Anderson M, Roshanravan H, Khine J and Dryer SE: Angiotensin II activation of TRPC6 channels in rat podocytes requires generation of reactive oxygen species. J Cell Physiol 229: 434-442, 2014.

29. Chen S, He FF, Wang H, Fang Z, Shao N, Tian XJ, Liu JS, Zhu ZH, Wang YM, Wang S, et al: Calcium entry via TRPC6 mediates albumin overload-induced endoplasmic reticulum stress and apoptosis in podocytes. Cell Calcium 50: 523-529, 2011
30. Zhang H, Ding J, Fan Q and Liu S: TRPC6 up-regulation in Ang II-induced podocyte apoptosis might result from ERK activation and NF-kappaB translocation. Exp Biol Med (Maywood) 234: 1029-1036, 2009

31. Schlöndorff J, Del Camino D, Carrasquillo R, Lacey V and Pollak MR: TRPC6 mutations associated with focal segmental glomerulosclerosis cause constitutive activation of NFAT-dependent transcription. Am J Physiol Cell Physiol 296: C558-C569, 2009.

32. Nijenhuis T, Sloan AJ, Hoenderop JG, Flesche J, van Goor H, Kistler AD, Bakker M, Bindels RJ, de Boer RA, Möller CC, et al: Angiotensin II contributes to podocyte injury by increasing TRPC6 expression via an NFAT-mediated positive feedback signaling pathway. Am J Pathol 179: 1719-1732, 2011.

33. Danda RS, Habiba NM, Rincon-Choles H, Bhandari BK, Barnes JL, Abboud HE and Pergola PE: Kidney involvement in a nongenetic rat model of type 2 diabetes. Kidney Int 68: 2562-2571, 2005

34. Graham S, Ding M, Sours-Brothers S, Yorio T, Ma JX and Ma R: Downregulation of TRPC6 protein expression by high glucose, a possible mechanism for the impaired $\mathrm{Ca} 2+$ signaling in glomerular mesangial cells in diabetes. Am J Physiol Renal Physiol 293: F1381-F1390, 2007.

35. Ilatovskaya DV, Palygin O, Levchenko V, Endres BT and Staruschenko A: The role of angiotensin II in glomerular volume dynamics and podocyte calcium handling. Sci Rep 7: 299, 2017.

36. Livak KJ and Schmittgen TD: Analysis of relative gene expression data using real-time quantitative PCR and the 2(-Delta Delta C(T)) method. Methods 25: 402-408, 2001

37. Su J, Li SJ, Chen ZH, Zeng CH, Zhou H, Li LS and Liu ZH Evaluation of podocyte lesion in patients with diabetic nephropathy: Wilms' tumor-1 protein used as a podocyte marker. Diabetes Res Clin Pract 87: 167-175, 2010.

38. Marshall SM: The podocyte: A potential therapeutic target in diabetic nephropathy? Curr Pharm Des 13: 2713-2720, 2007.

39. Wolf G, Chen S and Ziyadeh FN: From the periphery of the glomerular capillary wall toward the center of disease: Podocyte injury comes of age in diabetic nephropathy. Diabetes 54: 1626-1634, 2005

40. Menè P, Punzo G and Pirozzi N: TRP channels as therapeutic targets in kidney disease and hypertension. Curr Top Med Chem 13: 386-397, 2013.

41. Thilo F, Liu Y, Loddenkemper C, Schuelein R, Schmidt A, Yan Z, Zhu Z, Zakrzewicz A, Gollasch M and Tepel M: VEGF regulates TRPC6 channels in podocytes. Nephrol Dial Transplant 27: 921-929, 2012.

42. Weber EW, Han F, Tauseef M, Birnbaumer L, Mehta D and Muller WA: TRPC6 is the endothelial calcium channel that regulates leukocyte transendothelial migration during the inflammatory response. J Exp Med 212: 1883-1899, 2015.

43. Durvasula RV and Shankland SJ: Activation of a local renin angiotensin system in podocytes by glucose. Am J Physiol Renal Physiol 294: F830-F839, 2008.

44. Yoo TH, Li JJ, Kim JJ, Jung DS, Kwak SJ, Ryu DR, Choi HY, Kim JS, Kim HJ, Han SH, et al: Activation of the renin-angiotensin system within podocytes in diabetes. Kidney Int 71: 1019-1027, 2007.

45. Gabriel CH, Gross F, Karl M, Stephanowitz H, Hennig AF, Weber M, Gryzik S, Bachmann I, Hecklau K, Wienands J, et al: Identification of novel nuclear factor of activated $\mathrm{T}$ cell (NFAT)-associated proteins in T cells. J Biol Chem 291: 24172-24187, 2016

46. Pachulec E, Neitzke-Montinelli V and Viola JP: NFAT2 regulates generation of innate-like $\mathrm{CD} 8^{+} \mathrm{T}$ lymphocytes and $\mathrm{CD} 8^{+} \mathrm{T}$ lymphocytes responses. Front Immunol 7: 411, 2016.

47. Yang H, Zhao B, Liao C, Zhang R, Meng K, Xu J and Jiao J: High glucose-induced apoptosis in cultured podocytes involves TRPC6-dependent calcium entry via the RhoA/ROCK pathway. Biochem Biophys Res Commun 434: 394-400, 2013.

48. Li Z, Xu J, Xu P, Liu S and Yang Z: Wnt/ $\beta$-catenin signalling pathway mediates high glucose induced cell injury through activation of TRPC6 in podocytes. Cell Prolif 46: 76-85, 2013.

49. Qiu G and Ji Z: AngII-induced glomerular mesangial cell proliferation inhibited by losartan via changes in intracellular calcium ion concentration. Clin Exp Med 14: 169-176, 2014.

50. Lawrence MC, Bhatt HS and Easom RA: NFAT regulates insulin gene promoter activity in response to synergistic pathways induced by glucose and glucagon-like peptide-1. Diabetes 51: 691-698, 2002 
51. Nilsson J, Nilsson LM, Chen YW, Molkentin JD, Erlinge D and Gomez MF: High glucose activates nuclear factor of activated $\mathrm{T}$ cells in native vascular smooth muscle. Arterioscler Thromb Vasc Biol 26: 794-800, 2006.

52. Li R, Zhang L, Shi W, Zhang B, Liang X, Liu S and Wang W: NFAT2 mediates high glucose-induced glomerular podocyte apoptosis through increased Bax expression. Exp Cell Res 319: 992-1000, 2013
53. Wang L, Chang JH, Paik SY, Tang Y, Eisner W and Spurney RF: Calcineurin $(\mathrm{CN})$ activation promotes apoptosis of glomerular podocytes both in vitro and in vivo. Mol Endocrinol 25: 1376-1386, 2011

54. Zhang L, Li R, Shi W, Liang X, Liu S, Ye Z, Yu C, Chen Y, Zhang B, Wang W, et al: NFAT2 inhibitor ameliorates diabetic nephropathy and podocyte injury in $\mathrm{db} / \mathrm{db}$ mice. Br J Pharmacol 170: 426-439, 2013. 